\title{
CDISC SDTM Urinary System Test Name Terminology
}

National Cancer Institute

\section{Source}

National Cancer Institute. CDISC SDTM Urinary System Test Name Terminology. NCI

Thesaurus. Code C129941.

Terminology associated with the urinary system test name codelist of the Clinical Data Interchange Standards Consortium (CDISC) Study Data T abulation Model (SDT M). 